\title{
Optimized Reconstruction Algorithm Based Analysis of Effects of Exercise Therapy Combined with Celecoxib Capsules on the Rehabilitation of Knee Osteoarthritis Using Computed Tomography Images
}

\author{
Ying Shi $\mathbb{D}^{1},{ }^{1}$ Yongsheng Yang $\mathbb{D}^{2},{ }^{2}$ and Fengjun Wang $\mathbb{D}^{3}$ \\ ${ }^{1}$ Institute of Sports Rehabilitation, Baotou Medical College, Baotou 014060, China \\ ${ }^{2}$ Department of Rehabilitation Medicine and Physiotherapy, \\ The General Hospital of the Inner Mongolia Autonomous Region of the People's Armed Police Force, Hohhot 010040, China \\ ${ }^{3}$ Department of Acupuncture and Tuition of Mongolian Medicine, Hohhot Mongolian Hospital of Traditional Chinese Medicine, \\ Hohhot 010010, China \\ Correspondence should be addressed to Ying Shi; 102007196@btmc.edu.cn
}

Received 9 May 2021; Revised 9 June 2021; Accepted 18 June 2021; Published 28 June 2021

Academic Editor: Gustavo Ramirez

Copyright ( $\odot 2021$ Ying Shi et al. This is an open access article distributed under the Creative Commons Attribution License, which permits unrestricted use, distribution, and reproduction in any medium, provided the original work is properly cited.

\begin{abstract}
The aim was to explore the application value of computed tomography (CT) images based on optimized reconstruction algorithm (ORA) in knee osteoarthritis (KOA) and to analyze the rehabilitation effect of exercise therapy combined with celecoxib capsules on KOA. On the basis of iterative reconstruction algorithm, sparse data and compressed sensing (CS) algorithm were introduced in this study for the image reconstruction and were compared with the reconstruction effects of other algorithms. 105 patients with KOA were selected as the subjects investigated, who were treated in the hospital from December 2018 to January 2020. They were divided randomly into group A (exercise therapy), group B (celecoxib capsule therapy), and group C (exercise therapy combined with celecoxib capsules), with 35 patients in each group. All the patients were scanned by CT. Visual analogue score (VAS) method was adopted to compare the pain symptoms of patients in the three groups before and after treatment. The Lysholm knee function score was employed to evaluate the knee function of the patient before and after treatment, and finally the difference in the total effective rate of treatment was compared. The results showed that the reconstruction algorithm in this study had a higher degree of coincidence with the original image, and its root mean square error (RMSE), peak signal-to-noise ratio (PSNR), and structural similarity (SSIM) were greatly higher than those of filtered back-projection (FBP) algorithm. There was no huge difference between Lysholm score and VAS in patients of the three groups before treatment $(P>0.05)$. Besides, Lysholm score in all dimensions and its total score after treatment were higher than those of patients in the three groups before treatment $(P<0.05)$, and those of patients in group $\mathrm{C}$ increased extremely in contrast to group A $(P<0.05)$. VAS after treatment were all lower in patients of the three groups than those before treatment $(P<0.05)$, and VAS of patients in group $\mathrm{C}$ were sharply lower than those of group A $(P<0.05)$, but the total effective rate of patients in group $\mathrm{C}$ was dramatically higher than the rate of group $\mathrm{A}(P<0.05)$. It indicated that an algorithm with short scanning time and good image quality was established based on iterative reconstruction algorithm. Exercise therapy combined with celecoxib capsules could alleviate the pain symptoms and improve the function of knee joint, which was more advantageous than the simple rehabilitation comprehensive therapy. Therefore, it had higher clinical value for the patients with KOA.
\end{abstract}

\section{Introduction}

At present, methods applied in the clinical treatment of KOA mainly include oral drugs (non-steroid anti-inflammatory drugs and anti-inflammatory analgesics), articular cavity drug injection (sodium hyaluronate, etc.), and surgical treatment (total knee arthroplasty, arthroscopic cleanup, etc.) [1]. Oral medication can treat the clinical symptoms of 
patients in a short period of time but will cause adverse reactions such as gastrointestinal reactions [2], of which, celecoxib capsule is one of the drugs commonly used in the treatment of KOA disease, which can relieve the pain, joint stiffness, joint swelling/effusion, and other problems of the patient [3]. The effect of joint cavity drug injection is better than oral drugs, but its treatment cost is expensive and recurrence rate is also high [4]. Surgical treatment can improve markedly the clinical symptoms of patients, and the cure rate for osteoarthritis is high with the high cost, and there is a greater risk of treatment. Exercise therapy has been widely applied in the treatment of joint diseases. For example, Bartholdy et al. proved that muscle strength training for KOA patients could improve the knee extensor strength of patients [5]. Goh et al. found that exercise therapy could improve the pain and knee function of KOA patients [6]. Therefore, it is of great significance to find an economical and effective treatment method.

CT images can quantitatively and qualitatively evaluate the spatial morphology and location of the course of osteoarthritis [7]. The use of intelligent algorithms for medical image processing can improve the efficiency of clinical diagnosis. Hsieh et al. modified the Canny operator and CS algorithm to improve the edge blur during CT reconstruction [8]. Rajendran et al. built a spectral prior image constrained compressed sensing framework based on the modified CS algorithm and applied it to lung CT images to visualize lung nodules [9]. Thus, this study firstly applied ORA to reconstruct the CT images of patients and evaluated the therapeutic effect of oral celecoxib and exercise therapy for KOA disease based on clinical symptoms, which would provide an evidence for promoting the clinical therapeutic effect of KOA.

\section{Materials and Methods}

2.1. Subjects Investigated and Grouping. A total of $105 \mathrm{KOA}$ patients were selected as the subjects investigated including 63 males and 42 females, who were admitted to hospital from December 2018 to January 2020. Their age ranged from 55 to 67 years, and the average age was $59.82 \pm 5.56$ years. There were 83 patients with single knee joint disease and 22 patients with double knee joint disease. The course of disease was $0.25-12$ years, and the average course was $6.58 \pm 5.22$ years. The criteria for inclusion were defined to include patients who were diagnosed with KOA disease based on the criteria formulated by the American Rheumatism Association, suffered from osteoarthritis in the single or double knee joints, and were in stage 2 based on Kellgren-Lawrence classification. The criteria for exclusion were defined to contain patients who suffered from meniscus or cruciate ligament injury, had knee bone diseases such as rheumatism, took hormone drugs before treatment, suffered from severe organ dysfunction, and cannot cooperate with the treatment. This experiment had been approved by the Ethics Committee of the hospital. The enrolled patients and their family members had known the specific content of this experiment and signed the informed consent forms.
The volume and density of subpatellar fat pad of all patients were scanned by CT before and after treatment. Before the treatment, $105 \mathrm{KOA}$ patients enrolled in the study were divided randomly into an exercise therapy group (group A, $n=35$ ), a celecoxib group (group B, $n=35$ ), and an exercise therapy combined with celecoxib group (group C, $n=35$ ).

2.2. Optimized Reconstruction of Computed Tomography Images. Patients were in supine position, and their knees were put together. After that, Brilliance 64-row spiral CT (Philips, Netherlands) was employed to scan their subpatellar fat pads. The thickness of the scanning layer was $3 \mathrm{~mm}$, and the spacing was $1.3 \mathrm{~mm}$. The effective information in the obtained CT images could not be well utilized due to the influence of collection environment and collection angle. Therefore, the improved CS (ICS) was adopted to reconstruct the CT images of patients.

The matrix obtained by parallel beam scanning met the conditions of restricted isometry property (RIP) [10]. The images repaired by sparse signal had stable solutions. The signal applied a linear combination of multiple eigenvectors to represent sparse data. It was assumed that $X=\left[x_{1}, x_{2}, \ldots\right.$, $\left.x_{N}\right]^{T}$ and $X \in R^{N}$ ( $N$ was the length of one-dimensional discrete data). $X$ could be expressed through using a linear combination of orthogonal basis $L=\left[l_{1}, l_{2}, \ldots, l_{N}\right]$ and $N \in R^{N}$, as shown in the following equation:

$$
X=\sum_{i=1}^{N} \varphi_{i} a_{i}=\varphi a
$$

In equation (1), $a$ stood for $k<<N$ nonzero coefficient. After the sparse transformation, the sparse with signal length $S(S<<N)$ could be obtained as follows:

$$
\begin{aligned}
& y=\Phi x=\Phi \varphi a=\Theta a, \\
& \Theta=\Phi \varphi .
\end{aligned}
$$

In equation (2), $\Phi$ expressed a measurement matrix $(S<N)$ and $\varphi$ represented a sparse matrix.

The necessary and sufficient conditions for the above were that the vector $u$ and the measurement matrix $\Theta$ must meet the constraints of RIP adjustment, which could be calculated as follows.

$$
1-\alpha \leq\left(\frac{\|\Theta u\|_{2}}{\|u\|_{2}}\right) \leq 1+\alpha
$$

In equation (3), $\alpha>0$.

The algebraic reconstruction technique (ART) algorithm was regarded as the forward projection in most of the iteration process of CS, but the number of iterations of ART and the quality of the reconstructed image would be influenced by the projected image characteristics and quantity [11]. Therefore, this study adopted maximum likelihood expectation maximization (MLEM) algorithm to improve the CS, so as to obtain the improved CS for CT image reconstruction. 
The finite difference transform (FDT) was for the sparse transformation of two-dimensional image data because the finite difference of the generated image had a good sparse performance. Then, the total variation (TV) of the two-dimensional image could be defined as follows:

$$
\operatorname{TV}(F)=\sum_{u, v}\left(\sqrt{\left(f_{u, v}-f_{u-1, v}\right)^{2}}+\sqrt{\left(f_{u, v}-f_{u, v-1}\right)^{2}}\right) .
$$

The reconstructed image $F$ was obtained by solving the above equation, as shown in the following.

$$
\begin{array}{r}
\min \operatorname{TV}(F), \\
\text { s.t. } R=A F, \\
F \geq 0 .
\end{array}
$$

In equation (5), $R$ represents an image after transmission projection and $A$ stood for an observation matrix.

The ICS was for image reconstruction, and its specific process is shown in Figure 1. In each equation of Figure 1, $k$ stood for the total number of iterations, $f_{v}^{(k)}$ expressed the estimated value after $k^{\text {th }}$ iteration, $b_{u}$ represented the projected value of the $u^{\text {th }}$ ray, $p_{u, v}$ stood for the probability that the $v^{\text {th }}$ pixel of the object was detected by the detector $u$ unit, $N$ meant the ordinal number of iterations, $\beta$ represented the moderator factor, and $\alpha$ stood for an arbitrarily small positive number.

After reconstruction, the reconstruction effect was evaluated by root mean square error (RMSE), structural similarity (SSIM), and peak signal-to-noise ratio (PSNR).

$$
\begin{aligned}
\text { RMSE } & =\sqrt{\frac{1}{m, n} \sum_{i=0}^{m=1} \sum_{j=0}^{n=1}\|x(i, j)-y(i, j)\|^{2},} \\
\operatorname{SSIM}(X, Y) & =\frac{\left(2 u_{X} u_{Y}+C_{1}\right)\left(2 \delta_{X Y}+C_{2}\right)}{\left(u_{X}^{2} u_{Y}^{2}+C_{1}\right)\left(\delta_{X}^{2} \delta_{Y}^{2}+C_{2}\right)}, \\
\operatorname{PSNR} & =20 \cdot \log _{10}\left(\frac{\text { image }_{\max }}{\mathrm{MSE}}\right) .
\end{aligned}
$$

In equations (6)-(8), $x(i, j)$ and $X$ represented the original images; $y(i, j)$ and $Y$ expressed the reconstructed images; $u_{X}$ and $u_{Y}$ are the mean values of $X$ and $Y$, respectively; ${ }_{\delta}^{X}$ and ${ }_{\delta}^{Y}$ stood for the standard deviations of $X$ and $Y$, respectively; $\delta_{X Y}$ expressed the covariance of $X$ and $Y$; $C_{1}=(0.01 L)^{2}$ and $C_{2}=(0.03 L)^{2} ; L$ represented the dynamic range of the image; image max $_{\max }$ expressed the maximum value of the image; and MSE stood for the mean square error between the original image and the reconstructed image.

2.3. Therapeutic Methods. Exercise therapy for KOA included 5 methods as follows. The first one was supine isometric training. One patient took the supine position, extended the knee joints, slightly raised the knee joints, and tightened the muscles that maintained for 5 seconds. The second one was supine isotonic training. One patient was in the supine position and extended the knee joints. The contralateral knee joint was bent $90^{\circ}$, and the straight legs were raised to the level of the contralateral knee joints for $5 \mathrm{sec}$ onds. After completion, the knee joints were slowly restored to the initial position. The third one was prone isotonic training. One patient was in prone position, extended the knee joints, and fixed the thigh without moving, and the calves slowly flexed to the maximum extent for 5 seconds. After that, he or she slowly returned to the initial position. The fourth one was supine bridge closed chain movement. One patient was in the supine position. The feet on both sides and the back were considered as support points; he or she slowly lifted up the waist and hips that maintained for 5 seconds. After completion, he or she slowly returned to the initial position. The fifth one was upright lower limb closure movement. After one patient stood up, the knee joints flexed $90^{\circ}$ while squatting slowly for 5 seconds and then slowly returned to the initial position after completion. Each of the above 5 methods should be done 10 times, and the interval of each method was 30 seconds. Three of the above 5 methods were chosen to exercise twice a day (once in the morning and once in the afternoon), and exercise was for 4 days a week. After the patients were discharged from hospital, they needed to continue the above exercise.

Patients with KOA were treated with celecoxib capsules, and the specific treatment was shown as follows. One patient took celecoxib capsules orally once a day, and taking celecoxib capsules for 14 days consecutively was regarded as a course of treatment. The patient was required to take celecoxib capsules for at least 14 days to evaluate the therapeutic effect.

\subsection{Evaluation Indicators Based on Reconstructed Images of Computed Tomography. Based on the reconstructed CT images, the lower edge of patella and the $5 \mathrm{~mm}$ and $10 \mathrm{~mm}$ of the lower edge of patella were taken as axial positions for the layer measurement. Then, the tangents of the posterior edge of patellar ligament and the leading edge of bone were taken in turn to obtain the maximum distance of the inner edge of the medial and lateral synovium as the measurement results of the anterior, posterior, inner, and outer diameters. The plane of the central axis of patella in sagittal position was taken from reconstructed CT images, and the distance be- tween the middle point of lower patella and the highest tibial tubercle was detected as the measurement result of the diameter of lower patella. CT values of the lower edge of patella and $5 \mathrm{~mm}$ and $10 \mathrm{~mm}$ under the patella were taken as the average for density measurement results of the fat pad.}

2.5. Observation Indicators of Therapeutic Effect. VAS was employed to evaluate the pain degree of patients before treatment and after 30 days of treatment with different treatment methods. VAS scale divided it into $0-10$ based on the degree of pain, in which 10 meant the pain was unbearable and 0 stood for no pain. In addition, a score of $0-3$ indicated mild pain, which the patient could tolerate; a score of 4-6 represented moderate pain; namely, the pain affected the patient's sleep but was still tolerable; and a score of 7-10 showed severe pain, meaning the pain was unbearable. 


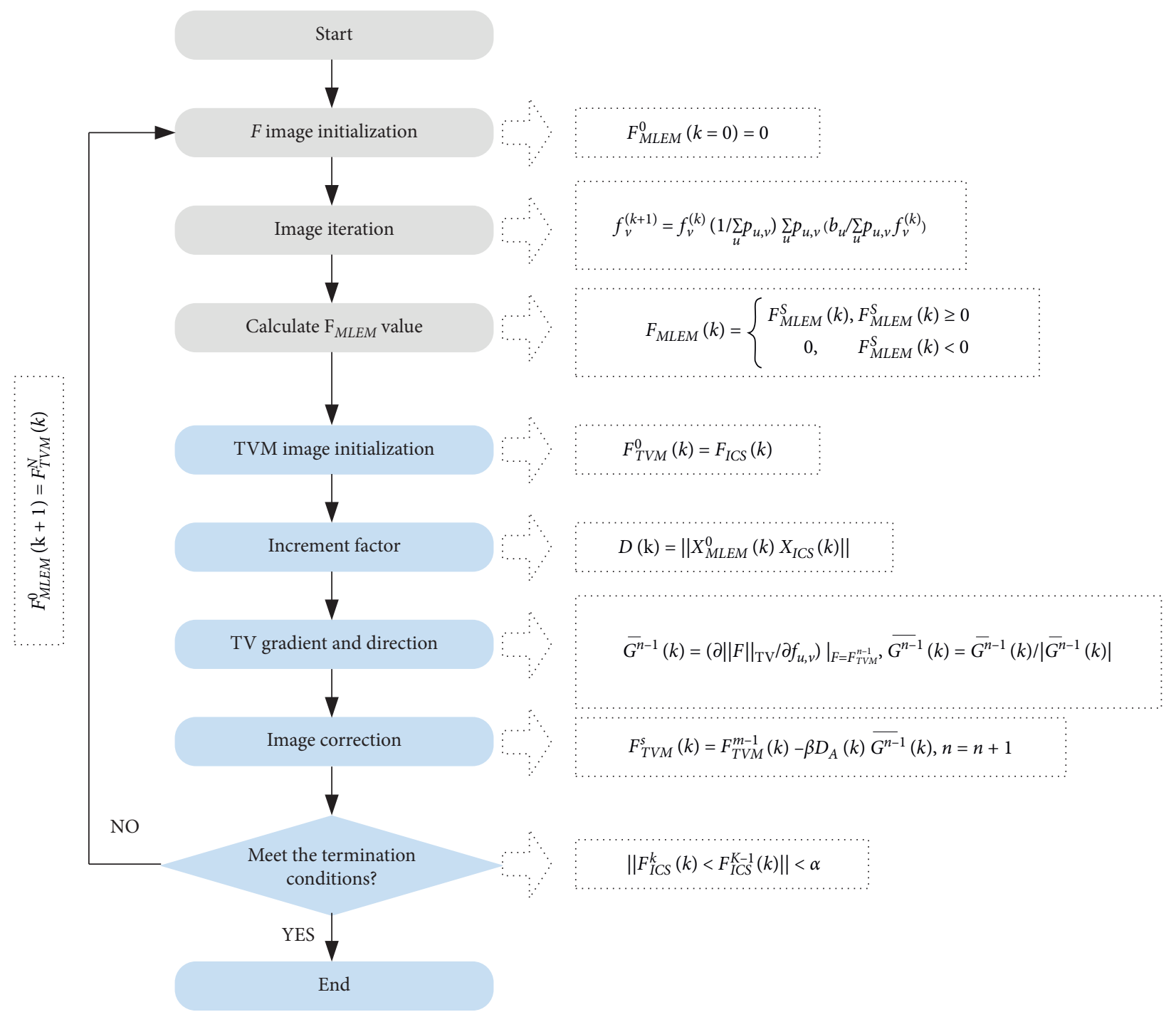

FIGURE 1: CT image reconstruction based on the ICS (note: the grey and blue module stood for a process of ICS with constraints and the total variation mode (TVM) process, respectively).

Lysholm knee joint function score scale was applied to evaluate the knee joint function of patients before and after 6 months of treatment with different treatment methods. Lysholm knee joint function rating scale contained a total of 8 evaluation items, with a total score of 100 points. When the score was below 70 points, it indicated that the knee joint function of the patient was affected obviously.

The effective rate was adopted to evaluate the therapeutic effect of different therapeutic methods. It was mainly grouped into obvious effect (the clinical symptoms of patients after treatment were improved greatly), effectivity (the clinical symptoms were promoted after treatment), no effect (the clinical symptoms after treatment were the same as those before treatment), and exacerbation (the clinical symptoms after treatment were more serious than those before treatment).

2.6. Statistical Methods. SPSS19.0 software was used for the statistics and processing of experimental data. Continuous variables were expressed as mean \pm standard deviation, and dichotomous variables were expressed as frequency or percentage (\%). The independent sample $t$-test was for the data that met the normal distribution to compare the differences among patients in the two groups, and the single factor variance analysis (ANOVA) was adopted to compare differences among patients in any two groups. When $P<0.05$, the difference was considered to be statistically significant.

\section{Results}

3.1. Comparison of the Basic Data among the Patients in the Three Groups. The age, sex ratio, number of diseased knees, course of disease, and body mass index (BMI) were collected in the patients of the three groups, and the differences in the above were compared. As shown in Table 1, there are no statistically obvious differences in age, sex ratio, number of diseased knees, BMI, and course of disease among patients in groups $\mathrm{A}, \mathrm{B}$, and $\mathrm{C}(P>0.05)$. 
TABLE 1: Comparison of general data in the patients from the three groups.

\begin{tabular}{|c|c|c|c|c|}
\hline Item & Group A $(n=35)$ & Group B $(n=35)$ & Group C $(n=35)$ & $P$ \\
\hline Age (years old) & $57.43 \pm 6.02$ & $58.22 \pm 7.15$ & $57.16 \pm 10.27$ & 0.325 \\
\hline Male patients (case/\%) & $19(18.10)$ & $21(20.00)$ & $23(21.90)$ & 0.093 \\
\hline Number of diseased knees & 43 & 41 & 43 & 0.317 \\
\hline BMI $\left(\mathrm{kg} / \mathrm{m}^{2}\right)$ & $23.15 \pm 2.11$ & $24.02 \pm 3.28$ & $23.09 \pm 2.19$ & 0.224 \\
\hline Course of disease (years) & $6.43 \pm 3.89$ & $6.50 \pm 5.17$ & $6.49 \pm 5.22$ & 0.449 \\
\hline
\end{tabular}

3.2. Comparison of Reconstruction Effects of Computed Tomography Images under Different Algorithms. In this study, FBP was regarded as a contrast. Firstly, FBP and ICS proposed in this study were compared to reconstruct the CT image of the knee joint of patients. Figure 2 indicates that both FBP and ICS could obtain the better reconstruction results, but the reconstructed image of ICS could more clearly display the useful information in the CT image.

In order to more accurately and clearly compare the differences in the original and reconstructed images in turn under different algorithms, this study took the section lines at the vertical and horizontal centers as objects for comparison. Figures 3(a) and 3(b) show that the profile lines in the horizontal center and vertical center of the reconstructed image by FBP are hugely different from the original image, indicating that there are many artifacts in the reconstructed image by FBP. The horizontal center and vertical center profile of ICS proposed in this study was very close to the original image.

Finally, the effects of different algorithms were quantitatively compared on the reconstruction of CT images. Table 2 reveals that the RMSE of the images reconstructed by ICS was sharply smaller than that of the images reconstructed by FBP. The PRSE and SSIM of ICS reconstructed images were extremely greater than those of FBP reconstructed images. The results showed that the ICS proposed in this study was superior to the FBP in image reconstruction.

\subsection{Comparison of Visual Analogue Score of Patients in the} Three Groups before and after Treatment. VAS in patients from each group are compared before treatment, as shown in Figure 4. The results indicated that VAS of patients in groups $\mathrm{A}, \mathrm{B}$, and $\mathrm{C}$ before treatment were not statistically substantial $\quad(t=0.224, \quad 0.241, \quad$ and 0.234 , and $P=0.239,0.212$, and 0.220 , respectively). VAS of patients in groups A, B, and C after 30 days of treatment were steeply lower than those before treatment $(t=8.393,8.834$, and 9.223 , and $P \leq 0.001$, respectively). VAS of patients in the three groups were compared 30 days after treatment, and it was found that there was no obvious difference in VAS among the patients in groups A and B $(t=0.220$ and $P=0.241$, respectively). VAS of patients in group C decreased sharply in contrast to groups A and B $(t=4.286$ and 3.339 , and $P=0.011$ and 0.026 , respectively).

3.4. Comparison of Knee Joint Function Assessment before and after Treatment among Patients in the Three Groups. Figure 5 shows the comparison of Lysholm knee function scores of patients in each group before treatment. The results indicated that there was no statistically extreme difference in Lysholm knee function scores in groups A, B, and C before treatment $\quad(t=0.209, \quad 0.211, \quad$ and 0.198 , and $P=0.310,0.248$, and 0.325 , respectively). After 30 days of treatment, Lysholm knee function scores of patients in groups $\mathrm{A}, \mathrm{B}$, and $\mathrm{C}$ were greatly higher than those before treatment $(t=10.449,10.586$, and 11.938 , and $P \leq 0.001$, respectively). After that, it was found that there was no statistically remarkable difference in Lysholm knee function scores among patients in groups A and B $(t=0.393$ and $P=0.116$, respectively), but Lysholm knee function scores in patients of group $\mathrm{C}$ were markedly higher than those of groups A and B $(t=5.324$ and 5.885, and $P=0.021$ and 0.035 , respectively).

3.5. Computed Tomography Measurement Results of Subpatellar Fat Pad before and after Treatment among Patients in the Three Groups. The comparison of the subpatellar fat pad between its upper and lower diameters of patients in each group before treatment is shown in Figure 6 . The results revealed that there was no statistically considerable difference in the subpatellar fat pad between the upper and lower diameters of patients from groups $\mathrm{A}, \mathrm{B}$, and $\mathrm{C}$ before treatment $\quad(t=0.328, \quad 0.334, \quad$ and 0.328 , and $P=0.239,0.221$, and 0.239 , respectively). After 30 days of treatment, the upper and lower diameters of subpatellar fat pad in groups A, B, and C were sharply lower than those before treatment $(t=4.223,4.509$, and 6.343, and $P=0.006,0.007$, and $P \leq 0.001$, respectively). Thus, it suggested that the difference among patients of groups $\mathrm{A}$ and $\mathrm{B}$ was not statistically substantial $(t=0.116$ and $P=0.097$, respectively), while the upper and lower diameters of subpatellar fat pad in patients of group $C$ were steeply smaller than those of groups A and B $(t=3.179$ and 3.516, and $P=0.015$ and 0.010 , respectively).

CT values of subpatellar fat pad in patients from each group are compared before treatment, as shown in Figure 7. The results disclosed that there was no statistically marked difference in the CT values of subpatellar fat pad among patients in groups A, B, and C before treatment $(t=0.198$, 0.116 , and 0.104 , and $P=0.368,0.407$, and 0.443 , respectively). After 30 days of treatment, CT values of subpatellar fat pad in groups A, B, and C increased greatly in contrast to before treatment $(t=8.198,8.094$, and 7.329, and $P \leq 0.001$, respectively). After that, it was found that there was no statistically substantial difference in the CT values of subpatellar fat pad among patients from groups $\mathrm{A}$ and $\mathrm{B}$ ( $t=0.218$ and $P=0.296$, respectively), while the CT value of subpatellar fat pad in patients from group $C$ reduced sharply 


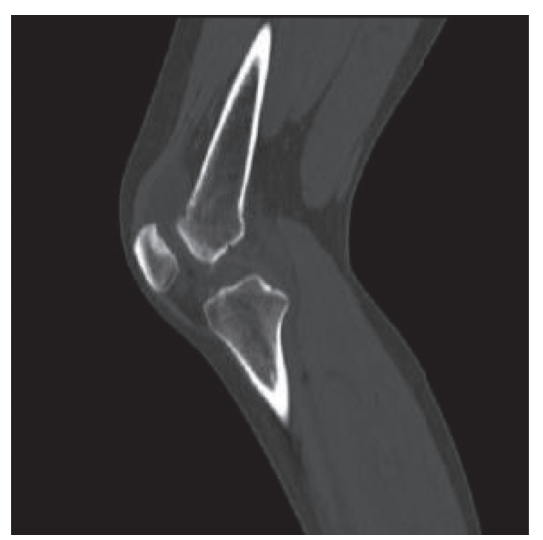

(a)

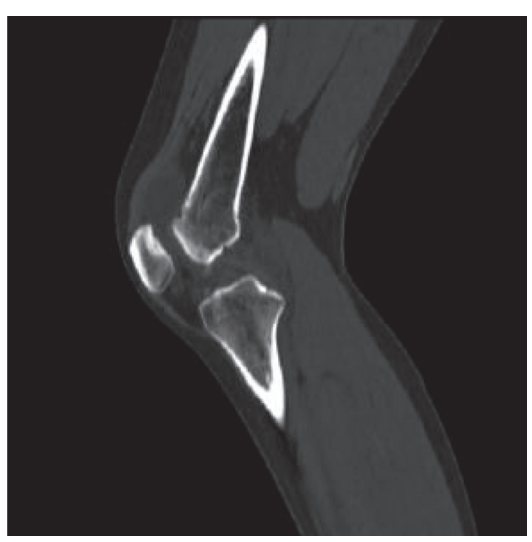

(b)

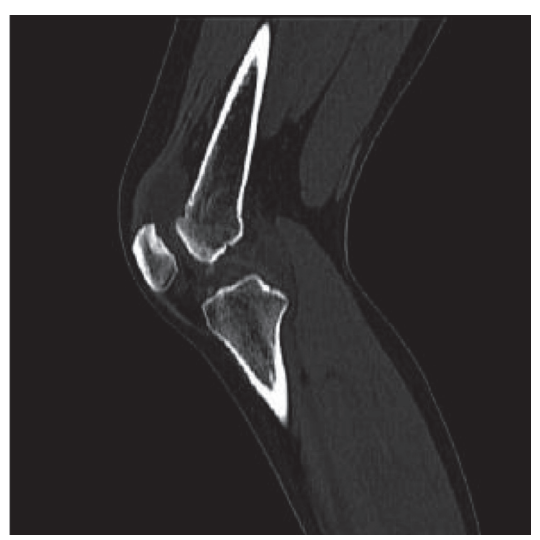

(c)

FIGURE 2: Results of CT image reconstruction with different algorithms (note: (a) the original CT image; (b) the CT image reconstructed by FBP; and (c) the CT image reconstructed by ICS).

TABLE 2: Quantitative evaluation of reconstructed images by different algorithms.

\begin{tabular}{lcc}
\hline Evaluation indicators & FBP & ICS \\
\hline RMSE & 9.233 & 3.432 \\
PSNR & 16.190 & 21.119 \\
SSIM & 0.282 & 0.884 \\
\hline
\end{tabular}

in contrast to groups A and B $(t=5.091$ and 4.883 , and $P=0.026$ and 0.034 , respectively).

3.6. Comparison of Clinically Therapeutic Effects among $\mathrm{Pa}$ tients in the Three Groups. Table 3 shows the comparison results of the clinical therapeutic effects with different treatment methods on KOA patients. There were no patients with exacerbation in groups $\mathrm{A}, \mathrm{B}$, and $\mathrm{C}$ after treatment. However, there were 9 invalid cases in group A, 8 invalid cases in group B, and 4 invalid cases in group $\mathrm{C}$. The results revealed that the total effective rate of patients in group $C$ was enormously higher than the rate of groups $\mathrm{A}$ and $\mathrm{B}$ ( $\chi^{2}=4.339$ and 3.626 , and $P=0.011$ and 0.023 , respectively).

\section{Discussion}

The manifestations of osteoarthritis hyperplasia, distortion, and swelling require comprehensive and detailed examination with imaging techniques. Spiral CT scanning of the patient's cartilage tissue and image reconstruction algorithm for CT image reconstruction can achieve the evaluation of the swelling degree of knee joint, ligament, and soft tissue $[12,13]$. Therefore, ICS was proposed in this study to reconstruct CT images of patients with KOA. The results showed that the RMSE, PSNR, and SSIM of the reconstructed images were smaller after reconstruction of ICS. Besides, RMSE can reflect the degree of difference among patients in each group, and the lower the value, the smaller the difference between reconstructed image and original image [14]. SSIM is employed to measure the structural similarity between graphs, and the larger the value, the more similar the reconstructed image to the original [15]. PSNR is applied to evaluate the image quality, and the larger the value, the lower the distortion degree of reconstructed image [8]. This disclosed that the algorithm proposed in this study was adopted to reconstruct the patient's original CT image and would not lose useful information of the original image, and the definition was higher.

Previous studies have shown that exercise therapy can improve patients' blood and lymph circulation and accelerate the repair speed of damaged cartilage by urging patients to exercise [5]. In this study, it was found that VAS could be reduced obviously and knee joint function of patients could be improved after exercise therapy by comparing with those before treatment $(P<0.05)$, which was consistent with the results of Goh et al. [6]. In addition, they discovered that exercise therapy could promote extremely the clinical treatment effects of patients with KOA. Moreover, it was observed that VAS, knee joint function, and effective rate of patients treated with exercise therapy combined with oral celecoxib capsules were dramatically better than those of patients treated with single treatment $(P<0.05)$, showing that oral medication combined with exercise therapy could markedly enhance the therapeutic effect of patients [16]. In addition, the changes of subpatellar fat pad were evaluated based on the reconstructed CT images before and after the treatment. The results suggested that the combination of exercise therapy and oral celecoxib capsules could reduce the upper and lower diameters of the subpatellar fat pad and increase the CT value. Therefore, exercise therapy combined with oral celecoxib capsules could slow down the increase rate of bone hypertrophy in the knee joint and reduce the severity of fat pad deformation $[17,18]$. 
TABle 3: Comparison of clinical therapeutic effects of different treatment methods.

\begin{tabular}{lccc}
\hline Therapeutic effect & Group A $(n=35)$ & Group B $(n=35)$ & Group C $(n=35)$ \\
\hline Obvious effect (cases/\%) & $17(48.57)$ & $18(51.43)$ & $21(60.00)$ \\
Effectivity (cases/\%) & $9(25.71)$ & $9(25.71)$ & $10(28.57)$ \\
No effect (cases/\%) & $9(25.71)$ & $8(22.86)$ & $4(11.43)$ \\
Exacerbation (cases/\%) & $0(0.00)$ & $0(0.00)$ & $0(0.00)$ \\
Total effective rate (cases/\%) & $26(74.29)$ & $27(77.14)$ & $31(88.57)^{*} \#$ \\
\hline
\end{tabular}

${ }^{*}$ The difference was statistically obvious compared to group A, $P<0.05 .{ }^{*}$ The difference was statistically substantial compared with group $\mathrm{B}, P<0.05$.

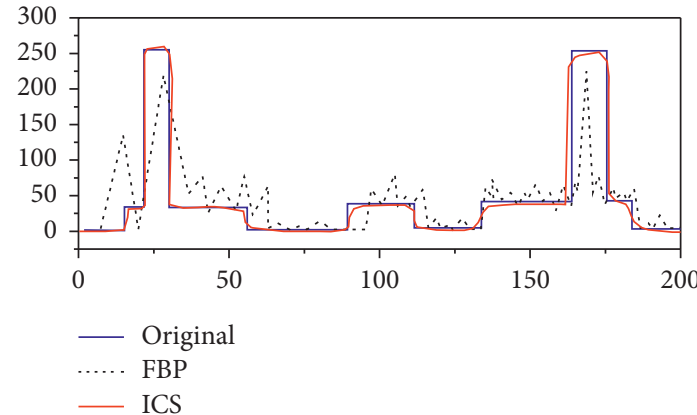

(a)

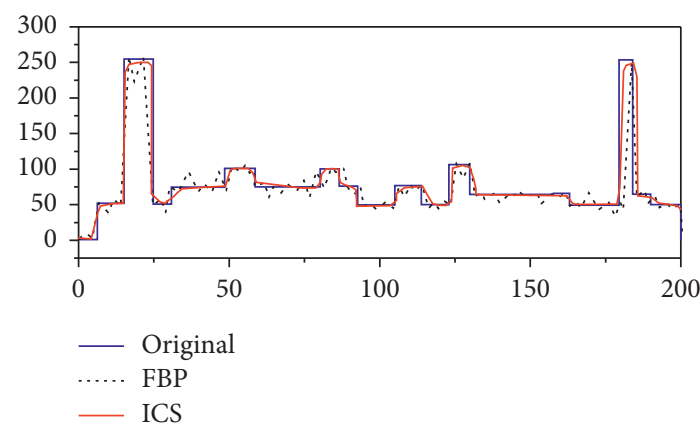

(b)

FIgURE 3: Comparison of central section lines in different directions of CT images reconstructed by different algorithms (note: (a) the comparison of the central section lines in the vertical direction and (b) the comparison of horizontal central section lines).

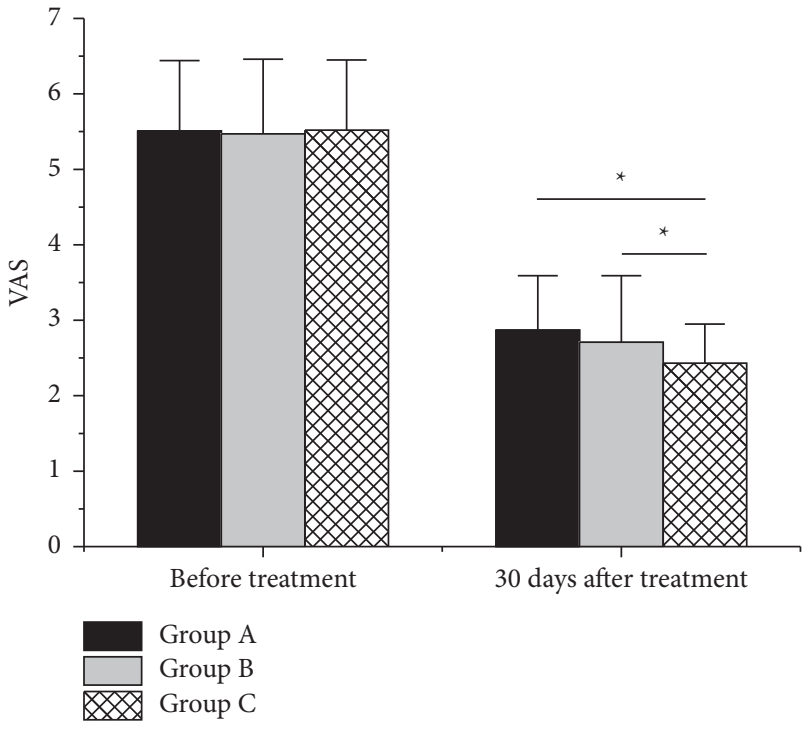

FIGURE 4: Comparison of VAS among patients in each group before and after treatment. $\left({ }^{*}\right.$ indicates that the difference between any two groups was statistically substantial, $P<0.05)$.

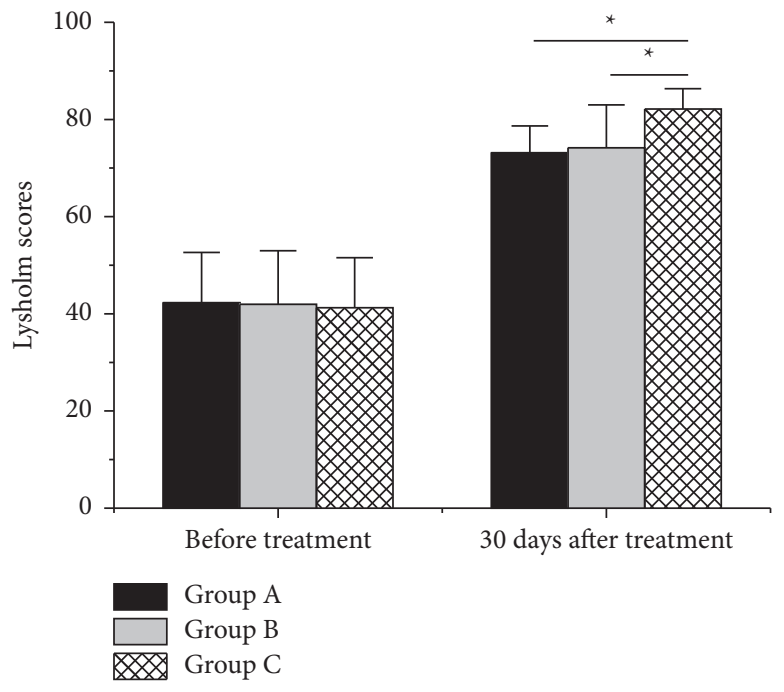

FIGURE 5: Comparison of Lysholm knee joint function scores among patients in each group before and after treatment. $\left({ }^{*}\right.$ indicates that the difference was statistically obvious among the patients in any of the two groups, $P<0.05$ ). 


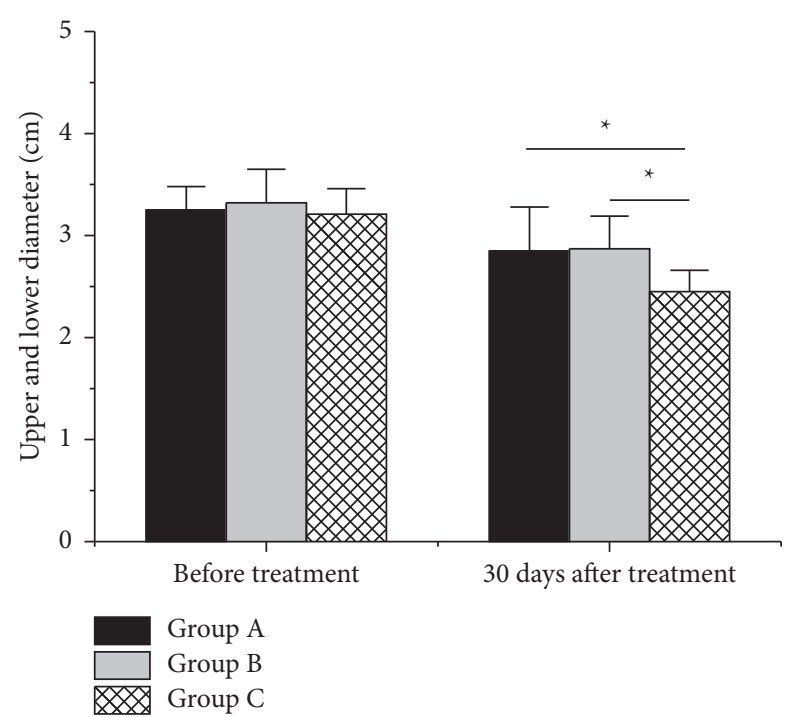

Figure 6: Comparison of the upper and lower diameters of subpatellar fat pad in patients of each group before and after treatment. ( ${ }^{*}$ indicates that the difference between any two groups was statistically substantial, $P<0.05$ ).

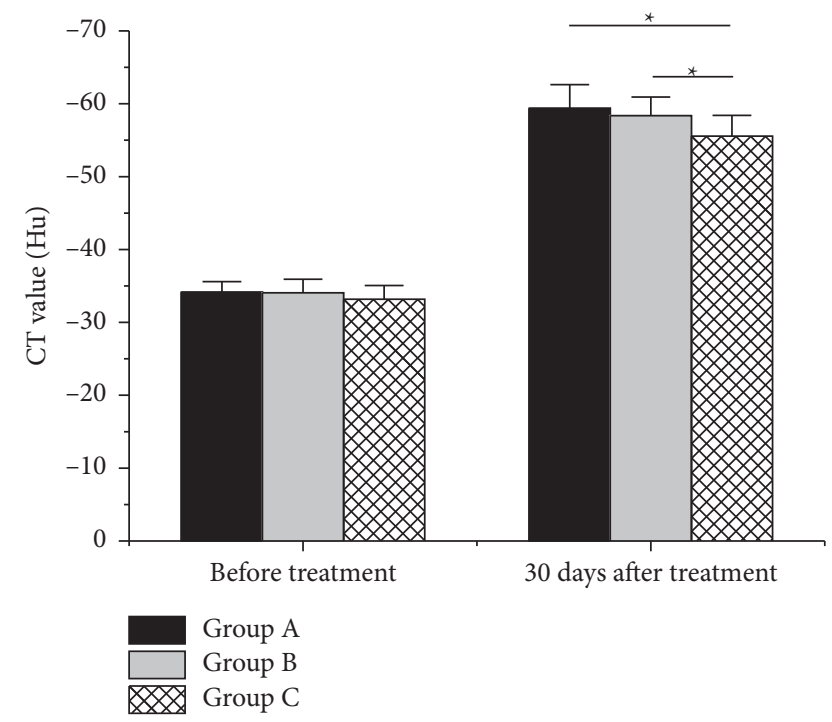

Figure 7: Comparison of CT values of subpatellar fat pad in patients from each group before and after treatment. ( ${ }^{*}$ indicates that the difference among the patients in any two groups was statistically significant, $P<0.05)$.

\section{Conclusion}

In this study, ICS was employed to reconstruct CT images of KOA patients, and the reconstruction effect was obviously better than the traditional algorithm. In clinical treatment, exercise therapy combined with oral celecoxib capsules could remarkably improve the patients' pain and knee joint function and can also slow down considerably the speed of knee joint fat pad deformation. However, the changes of the subpatellar fat pad in patients were only compared by CT before and after treatment. Thus, more CT indicators should be collected for comprehensive analysis later. The results of this study could provide evidence for promoting the clinical treatment effects of KOA disease.

\section{Data Availability}

No data were used to support this study.

\section{Conflicts of Interest}

The authors declare that they have no conflicts of interest.

\section{References}

[1] L. Arendt-Nielsen, O. Simonsen, M. B. Laursen et al., "Pain and sensitization after total knee replacement or nonsurgical treatment in patients with knee osteoarthritis: identifying potential predictors of outcome at 12 months," European Journal of Pain, vol. 22, no. 6, pp. 1088-1102, 2018.

[2] K. W. Lin, "Treatment of knee osteoarthritis," American Family Physician, vol. 98, no. 9, pp. 603-606, 2018.

[3] M. C. Hochberg, J. Martel-Pelletier, J. Monfort et al., "Combined chondroitin sulfate and glucosamine for painful knee osteoarthritis: a multicentre, randomised, double-blind, non-inferiority trial versus celecoxib," Annals of the Rheumatic Diseases, vol. 75, no. 1, pp. 37-44, 2016.

[4] L. Hangody, R. Szody, P. Lukasik et al., "Intraarticular injection of a cross-linked sodium hyaluronate combined with triamcinolone hexacetonide (cingal) to provide symptomatic relief of osteoarthritis of the knee: a randomized, doubleblind, placebo-controlled multicenter clinical trial," Cartilage, vol. 9, no. 3, pp. 276-283, 2018.

[5] C. Bartholdy, C. Juhl, R. Christensen et al., "The role of muscle strengthening in exercise therapy for knee osteoarthritis: a systematic review and meta-regression analysis of randomized trials," Seminars in Arthritis and Rheumatism, vol. 47, no. 1, pp. 9-21, 2017.

[6] S. L. Goh, M. S. M. Persson, J. Stocks et al., "Efficacy and potential determinants of exercise therapy in knee and hip osteoarthritis: a systematic review and meta-analysis," Annals of Physical and Rehabilitation Medicine, vol. 62, no. 5, pp. 356-365, 2019.

[7] R. De Laroche, E. Simon, N. Suignard et al., "Clinical interest of quantitative bone SPECT-CT in the preoperative assessment of knee osteoarthritis," Medicine, vol. 97, no. 35, Article ID e11943, 2018.

[8] C. J. Hsieh, T. K. Huang, T. H. Hsieh et al., "Compressed sensing based CT reconstruction algorithm combined with modified canny edge detection," Physics in Medicine and Biology, vol. 63, no. 15, Article ID 155011, 2018.

[9] K. Rajendran, S. Tao, D. Abdurakhimova et al., "Ultra-High resolution photon-counting detector CT reconstruction using spectral prior image constrained compressed-sensing (UHRSPICCS)," Proceedings of SPIE-The International Society for Optical Engineering, vol. 10573, Article ID 1057318, 2018.

[10] A. S. Charles, H. L. Yap, and C. J. Rozell, "Short-term memory capacity in networks via the restricted isometry property," Neural Computation, vol. 26, no. 6, pp. 1198-1235, 2014.

[11] F. M. Arrabal-Campos, L. M. Aguilera-Sáez, and I. Fernández, "Algebraic reconstruction technique for diffusion NMR experiments. application to the molecular weight prediction of polymers," The Journal of Physical Chemistry A, vol. 123, no. 4, pp. 943-950, 2019. 
[12] M. Stille, M. Kleine, J. Hägele et al., "Augmented likelihood image reconstruction," IEEE Transactions on Medical Imaging, vol. 35, no. 1, pp. 158-173, 2016.

[13] N. Magarelli, V. De Santis, G. Marziali et al., "Application and advantages of monoenergetic reconstruction images for the reduction of metallic artifacts using dual-energy CT in knee and hip prostheses," Radiologia Medica, vol. 123, no. 8, pp. 593-600, 2018.

[14] C. Jiang, Q. Zhang, R. Fan et al., "Super-resolution CT image reconstruction based on dictionary learning and sparse representation," Scientific Reports, vol. 8, no. 1, Article ID 8799, 2018.

[15] R. M. S. Joemai and J. Geleijns, "Assessment of structural similarity in CT using filtered backprojection and iterative reconstruction: a phantom study with $3 \mathrm{D}$ printed lung vessels," British Journal of Radiology, vol. 90, no. 1079, Article ID 20160519, 2017.

[16] J. Dunning, R. Butts, I. Young et al., "Periosteal electrical dry needling as an adjunct to exercise and manual therapy for knee osteoarthritis," The Clinical Journal of Pain, vol. 34, no. 12, pp. 1149-1158, 2018.

[17] M. de Rooij, M. van der Leeden, J. Cheung et al., "Efficacy of tailored exercise therapy on physical functioning in patients with knee osteoarthritis and comorbidity: a randomized controlled trial," Arthritis Care \& Research, vol. 69, no. 6, pp. $807-816,2017$.

[18] A. Nazari, A. Moezy, P. Nejati et al., "Efficacy of high-intensity laser therapy in comparison with conventional physiotherapy and exercise therapy on pain and function of patients with knee osteoarthritis: a randomized controlled trial with 12week follow up," Lasers in Medical Science, vol. 34, no. 3, pp. 505-516, 2019. 